\title{
Post-treatment serum lactic dehydrogenase as a predictive indicator for distant metastasis and survival of patients with nasopharyngeal carcinoma
}

\author{
Jin Wang ${ }^{1, *}$, Li Li ${ }^{2, *}$, Bai-qiang Dong ${ }^{1}$, Yu-jin Xu${ }^{1}$, Yuan-da Zheng ${ }^{1}$, Zhong-wen Sun ${ }^{3}$, \\ Yang Yang ${ }^{1}$, Yuan-Yuan Chen ${ }^{1}$, Xiao-zhong Chen ${ }^{1}$, Ming Chen ${ }^{1}$ \\ ${ }^{1}$ Department of Radiation Oncology, Zhejiang Key Lab of Radiation Oncology, Zhejiang Cancer Hospital, Hangzhou, People's \\ Republic of China \\ ${ }^{2}$ Department of Ultrasonography, Zhejiang Cancer Hospital, Hangzhou, People's Republic of China \\ ${ }^{3}$ Department of Oncology, Jining First People's Hospital, Jining, People's Republic of China \\ *These authors have contributed equally to this work
}

Correspondence to: Yuan-Yuan Chen, e-mail: Chenyy@zjcc.org.cn

Keywords: serum lactic dehydrogenase, intensity-modulated radiation therapy, nasopharyngeal carcinoma, prognostic factor, metastasis

Received: January 04, 2016

Accepted: March 18, 2016

Published: March 30, 2016

\section{ABSTRACT}

Purpose: To examine the function of serum lactic dehydrogenase (SLDH) level after intensity-modulated radiotherapy (IMRT) as a predictive factor for and locoregional relapse free survival (LRFS), distant metastasis-free survival (DMFS), disease free survival (DFS), and overall survival(OS) among patients with in-situ nasopharyngeal carcinoma (NPC).

Results: Compared with the normal pt-SLDH group, elevated pt-SLDH demonstrated significant lower DMFS (46 versus 66 months, hazard ratio (HR) $4.07,95 \%$ CI 2.43-6.80, $p<0.001$ ), DFS (46 versus 63 months, HR $2.78,95 \%$ CI 1.70-4.53, $p<0.001$ ), and OS (54 versus 66 months, HR 2.93, 95\% CI 1.65-5.23, $p<0.001)$. Distant metastasis were observed in $32.8 \%(20 / 61)$ patients with elevated pt-SLDH, and $8 \%(54 / 678)$ in normal SLDH (odds ratio (OR) 6.13, 95\% CI 3.35-11.18, $p<0.001)$. COX regression showed that pt-SLDH was an independent prognostic factors for OS (HR 2.91, 95\% CI 1.57-5.41, p < 0.001), DMFS (HR 4.21, 95\% CI 2.51-7.07, $p<0.001$ ), LRFS (HR 2.53, 95\% CI 1.22-5.24, $p<0.001$ ), and DFS (HR $2.81,95 \%$ CI 1.72-4.59, $p<0.001$ ).

Materials and Methods: The records of 739 in-situ NPC patients admitted to Zhejiang Cancer Hospital between January 2007 and May 2012 were retrospectively reviewed. The relationships between post-treatment SLDH (pt-SLDH) and LRFS, DMFS, DFS, and OS were analyzed.

Conclusions: Our finding indicated that elevated pt-SLDH could be a simple available prognostic indicator for distant metastasis and survival for in-situ NPC patients.

\section{INTRODUCTION}

Nasopharyngeal carcinoma (NPC) has been considered as a rare type of cancer globally yet it is relatively frequent in some regions, including some locations of South-Eastern Asia and a number of provinces in South-Eastern China. Intensity-modulated radiotherapy (IMRT), which can deliver high radiation dose to the tumor but render little radiation to surrounding tissues, is becoming a standard radiotherapy (RT) technique for NPC $[1,2]$. It is increasingly reported that IMRT alone or in combination with chemotherapy could improve locoregional control and overall survival. However, $19 \%$ to $29 \%$ of patients have been found to have distant metastasis with controlled loco-regional lesion [3, 4]. To date, distant metastasis is still the major cause of mortality among NPC patients [5]. It is desirable to identify patients with higher risk at distant metastasis and unfavorable prognosis. 
Lactate dehydrogenase (LDH), which reversibly transforms pyruvate to lactate under hypoxic conditions, is a tumor product in cancer patients. Serum LDH (SLDH) has also shown to be predictive of treatment outcome in various types of tumors $[6,7]$. This is probably associated with hypoxia-mediated radio-resistance and the upregulation of metastasis-associated genes [6-8]. Multiple studies reported that SLDH prior to treatment was a predictive indicator for survival or distant metastasis among patients with NPC [8-17]. However, very limited research had been performed to explore the clinical significance of SLDH during follow-up period.

The objective of this study was to examine the relationship between SLDH measured during the follow up period (pt-SLDH) and locoregional relapse free survival (LRFS), distant metastasis-free survival (DMFS), and overall survival (OS) in patients with in situ NPC after IMRT.

\section{RESULTS}

\section{Patient characteristics}

The characteristics of the patients including age, gender, pathologic type according to the World Health Organization (WHO) classification, and AJCC stage distribution were outlined in Table 1. Totally 739 patient records were included in the study. The median age of the patients was 49 years old ranging from 18 to 81 . The median overall RT treatment time was 44 days, and $84.8 \%$ of patients completed RT within 7 weeks. Six hundred and eighty-six patients received combined cisplatin-based concurrent chemotherapy. Six hundred and seventy-two patients received induction chemotherapy. One hundred and eighty-seven patients received adjuvant chemotherapy.

\section{Overall survival}

The follow-up time ranged from 3 to 72 months (median 34 months). The cumulative survive rate of 1, 3, 5 year was $97 \%, 92 \%, 81 \%$, respectively (Figure 1A). The LRFS rate at 1, 3, 5 year was $99 \%, 93 \%, 92 \%$, respectively (Figure 1B). The DMFS rate at 1,3,5 year was 97\%, $88 \%, 82 \%$, respectively (Figure 1C). The DFS rate at 1 , 3,5 year was $96 \%, 84 \%, 78 \%$, respectively (Figure 1D).

\section{Correlation of pt-SLDH with prognosis}

The average peak pt-SLDH level was $204.9 \pm 161.3 \mathrm{U} / \mathrm{L}$ ranging from $91-3278 \mathrm{U} / \mathrm{L}$. Patients with elevated pt-SLDH ( $>240 \mathrm{U} / \mathrm{L}$ ) demonstrated a significant lower OS compared to those with normal pt-SLDH $(<=240 \mathrm{U} / \mathrm{L})(54$ months versus 66 months, HR 2.93, $95 \%$ CI 1.65-5.23, $p<0.001$, Figure 2A). Also, significant shorter LRFS (60 months versus 68 months, HR 2.49, 95\% CI 1.21-5.16, $p=0.011$, Figure 2C), DMFS (46 months versus 66 months, HR 4.07, 95\% CI 2.43-6.80, $p<0.001$, Figure 2C), and DFS (46 months versus 63 months, HR $2.78,95 \%$ CI $1.70-4.53, p<0.001$, Figure 2D) were found in elevated pt-SLDH group compared to the normal group.

We performed another analysis by dividing the patients into two groups based on the median ptSLDH level of $205.0 \mathrm{U} / \mathrm{L}$. We found that the group of $\leq 205.0 \mathrm{U} / \mathrm{L}$ had significant prolonged median OS (67 months versus 61 months, HR 2.00, 95\% CI 1.09-3.53, $p=0.021$, Figure 3A), median DMFS ( 68 months versus 57 months, HR $2.84,95 \%$ CI $1.69-4.78 p<0.001$ Figure $3 \mathrm{C}$ ), and median DFS (64 months versus 56 months, HR 1.60, 95\% CI 1.03-2.48 $p=0.035$ Figure 3D) compared with the group of $>205.0 \mathrm{U} / \mathrm{L}$. However, no significant difference in RFS was found between the two groups (median: 68 months versus 66 months, $p=0.864$, Figure. 3B).

\section{Correlation between the SLDH change before and after the treatment with prognosis}

The average SLDH level before treatment was $175.7 \pm 43.11 \mathrm{U} / \mathrm{L}$ (ranging from 9-528 U/L), which was independent of patient's age, gender, $\mathrm{T}$ stage, $\mathrm{N}$ stage, and AJCC stage status. Univariate analysis found that pretreatment SLDH has no significant correlation with survival rate. However, we found that patients with normal pre-treatment SLDH and elevated pt-SLDH had poor OS, LRFS, DMFS, DFS (Figure 4).

We further analyzed the effect of the variance of SLDH between pre- and post- treatment $\left(\mathrm{V}_{\mathrm{SLDH}}\right)$. The $\mathrm{V}_{\mathrm{LDH}}$ from the 739 patients ranged from -381 to 3071 (Mean $28.51 \pm 168.95$ ). Then we rounded the mean value and used it to separate the patients into two groups: $\mathrm{V}_{\mathrm{SLDH}}>29 \mathrm{U} / \mathrm{L}$ and $\leq 29 \mathrm{U} / \mathrm{L}$. The group with $\mathrm{V}_{\text {SLDH }}>29 \mathrm{U} / \mathrm{L}$ had significant lower median OS (61 months vs 67 months, HR 1.87, 95\% CI 1.00-3.51, $p=0.047$ ), DMFS (56 months vs 67 months, HR 3.09, $95 \%$ CI $1.78-5.38, p<0.001$ ), and DFS (55 months vs 64 months, HR 1.93, 95\% CI 1.21-3.08, $p=0.005$ ) than those with VSLDH $\leq 29 \mathrm{U} / \mathrm{L}$, but there is no significant deference in LRFS between two groups ( $p=0.178$, Figure 5).

\section{Correlation of pt-SLDH with metastasis}

Distant metastases were found in 20 of the 61 (32.8\%) patients with elevated pt-SLDH, and in 54 of the $678(8 \%)$ patients with normal pt-SLDH (odds ratio (OR): 6.13, 95\% CI: $3.35-11.18, p<0.001)$.

Elevated pt-SLDH were found in 20 of 74 (27.0\%) patients who developed metastasis during follow-up, among whom $20 \%$ patients were found to have elevated pt-SLDH before metastasis were diagnosed. 
Table 1: Patient characteristics $(n=739$ patients $)$

\begin{tabular}{|l|l|}
\hline \multicolumn{1}{|c|}{ Characteristics } & \multicolumn{1}{|c|}{ No of patients (\%)* } \\
\hline Age, years & $415(55.1)$ \\
\hline$\leq 50$ & $324(43.8)$ \\
\hline >50 & \\
\hline Gender & $515(69.7)$ \\
\hline Male & $224(30.3)$ \\
\hline Female & \\
\hline Pathologic type (WHO 1992) & $65(8.8)$ \\
\hline I & $674(91.2)$ \\
\hline II and III & \\
\hline T category (AJCC 2009) & $68(9.2)$ \\
\hline T1 & $141(19.1)$ \\
\hline T2 & $331(44.8)$ \\
\hline T3 & $199(26.9)$ \\
\hline T4 & \\
\hline N category (AJCC 2009) & $79(10.7)$ \\
\hline N0 & $221(30.7)$ \\
\hline N1 & $351(47.5)$ \\
\hline N2 & $82(11.1)$ \\
\hline N3(N3a and N3b) & \\
\hline Stage (AJCC 2009) & $9(1.2)$ \\
\hline I & $78(10.6)$ \\
\hline II & $396(53.6)$ \\
\hline III & $174(23.5)$ \\
\hline IVa & $82(11.1)$ \\
\hline IVb & (A) \\
\hline
\end{tabular}

Abbreviations: WHO, World Health Organization; AJCC, American Joint Committee on Cancer.

*Value may not add to $100 \%$ because of rounding.

\section{Multivariate analysis}

COX regression showed that age, overall RT time, pt-SLDH, and tumor stage were independent influence factors for overall survival (Table 2). In multivariate analysis of DMFS, LRFS, DFS, elevated pt-SLDH was associated with a high risk of distant metastasis (HR 4.21 95\% CI 2.51-7.07 $p<0.001$ ), relapse (HR 2.53 95\% CI $1.22-5.24 p<0.001$ ), and disease progression (HR 2.81 $95 \%$ CI $1.72-4.59 p<0.001)$.

\section{DISCUSSION}

Our previous studies showed that inhibition of LDH induced G2/M cycle arrest, apoptosis, and autophagy in NPC and NSCLC cells $[18,19]$. In this study, we investigated the prognostic value of pt-SLDH for NPC patients through retrospective analysis of clinical data. We found that pt-SLDH was correlated significantly with risk of distant metastasis, survival time, and local relapse, especially when pt-SLDH was 29 U/L or higher than pretreatment level. Also, significant lower DMFS, DFS, OS were observed in patients with elevated pt-SLDH than those of normal pt-SLDH, disregard of the pretreatment SLDH level. Furthermore, elevated pt-SLDH was associated with high rate of distant metastasis, and could be a predictor for metastasis before it was identified. Our study indicated that pt-SLDH might be a good marker for monitoring the disease progress and treatment efficacy.

Our result was echoed by some other studies that indicated pt-SLDH being linked with failure of radiotherapy and chemotherapy in sarcomas, lymphomas, and carcinomas [8]. Jin et al. also observed poor survival in metastatic NPC patients with normal pre-treatment and 
Table 2: COX regression of clinical factor on overall survival (739 patients)

\begin{tabular}{|l|l|l|l|l|l|l|}
\hline Parameters & B & S.E & Wald & \multicolumn{1}{c}{ Exp (B) } & 95 \% CI \\
\hline Stage & 0.436 & 0.217 & 4.056 & $\mathbf{0 . 0 4 4}$ & 1.547 & $1.012-2.365$ \\
\hline pt-SLDH $\geq 240 \mathrm{U} / \mathrm{L}$ & 1.123 & 0.313 & 12.849 & $<\mathbf{0 . 0 0 1}$ & 3.074 & $1.684-5.680$ \\
\hline RT time & 0.058 & 0.020 & 8.323 & $\mathbf{0 . 0 0 4}$ & 1.06 & $1.019-1.103$ \\
\hline Age & 0.050 & 0.013 & 13.839 & $<\mathbf{0 . 0 0 1}$ & 1.051 & $1.024-1.079$ \\
\hline
\end{tabular}
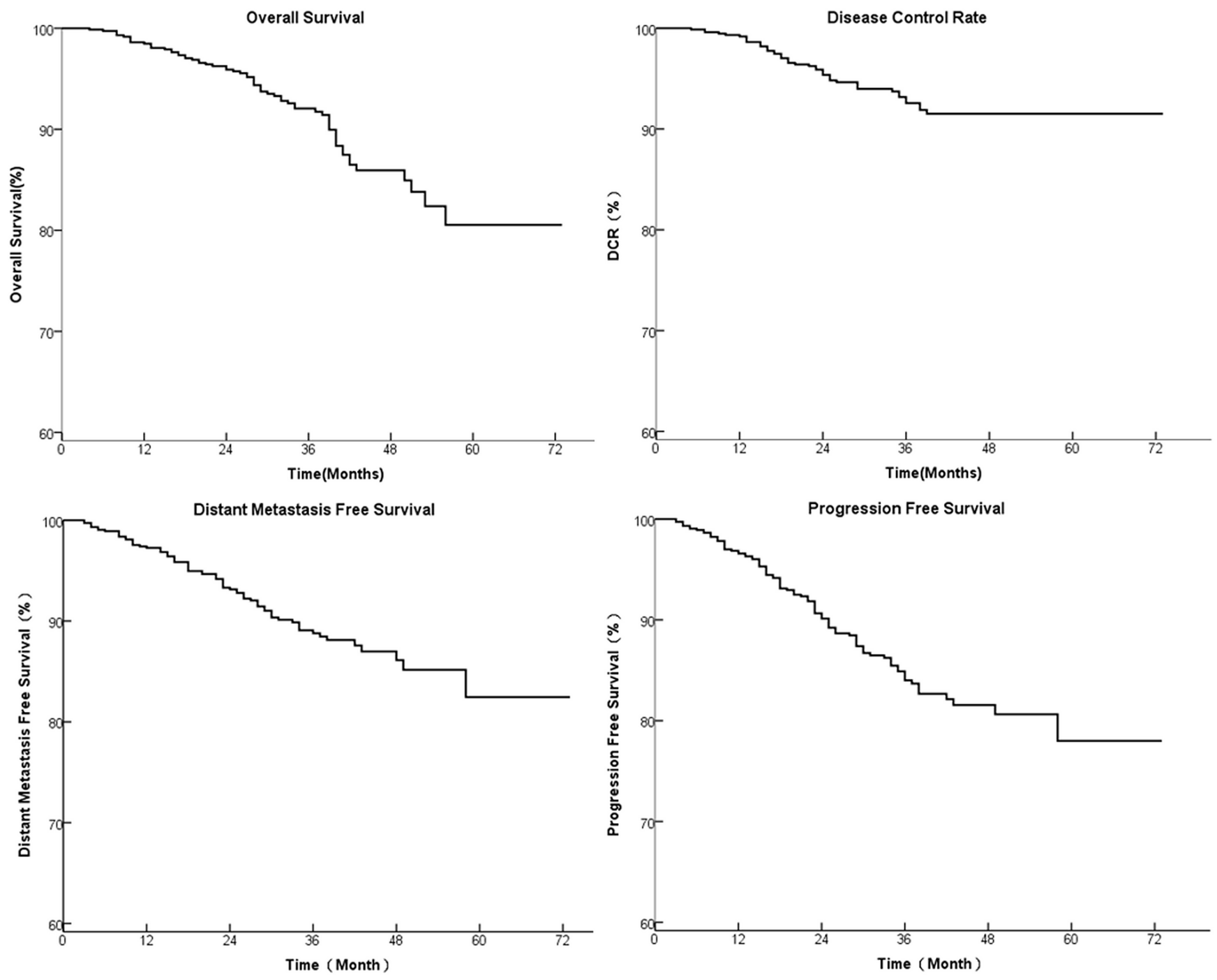

Figure 1: The 1, 3, 5 year survival of 739 patients with stage I to IVb NPC treated by IMRT. (A): OS, (B): LRFS, (C): DMFS, and (D): DFS. 
elevated pt-SLDH [5]. The SLDH level decreased while patients were in remission, and increased while patients were in relapse.

Many studies showed that SLDH had been identified as a prognostic indicator for NPC patients [9-15]. In locally advanced NPC patients, elevated pretreatment SLDH has been reported as a risk factor for distant metastasis, or local relapse $[8,10,16]$. Wan et al. analyzed a phase III randomized controlled study and found that
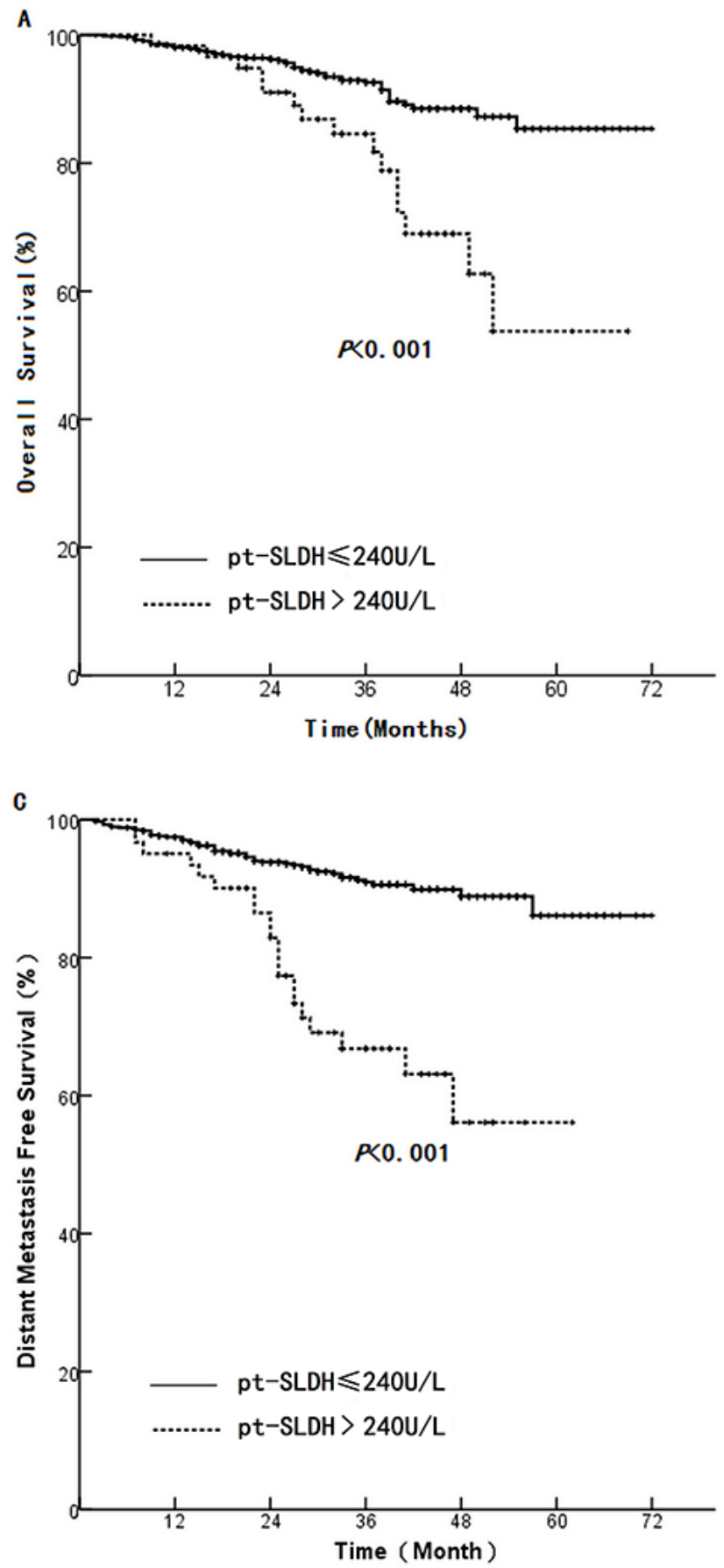

elevated pretreatment SLDH correlated with poor OS, DFS, LRFS, and DMFS in patients with locally advanced NPC [17]. Zhang et al. found that pre-treatment SLDH could be prognostic for OS and DMFS in NPC patients with a positive family history [20].

Unfortunately, there is only a few publications about the pt-SLDH. Our study is the only study with largest case number on prognostic value of pt-SLDH for in-situ NPC. The mechanisms of the observation in this

B

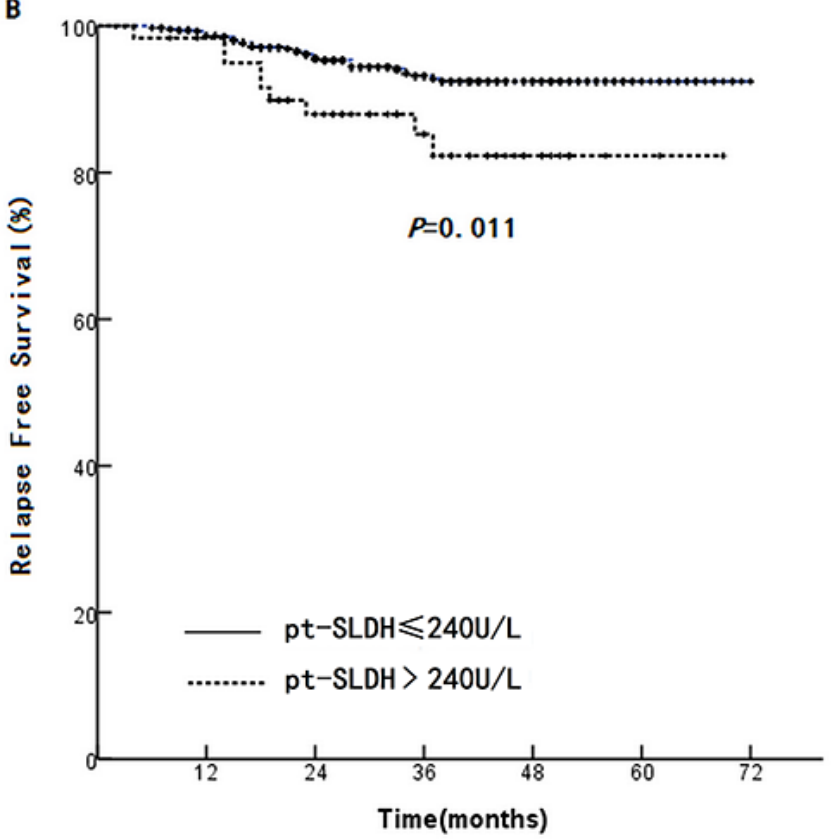

D

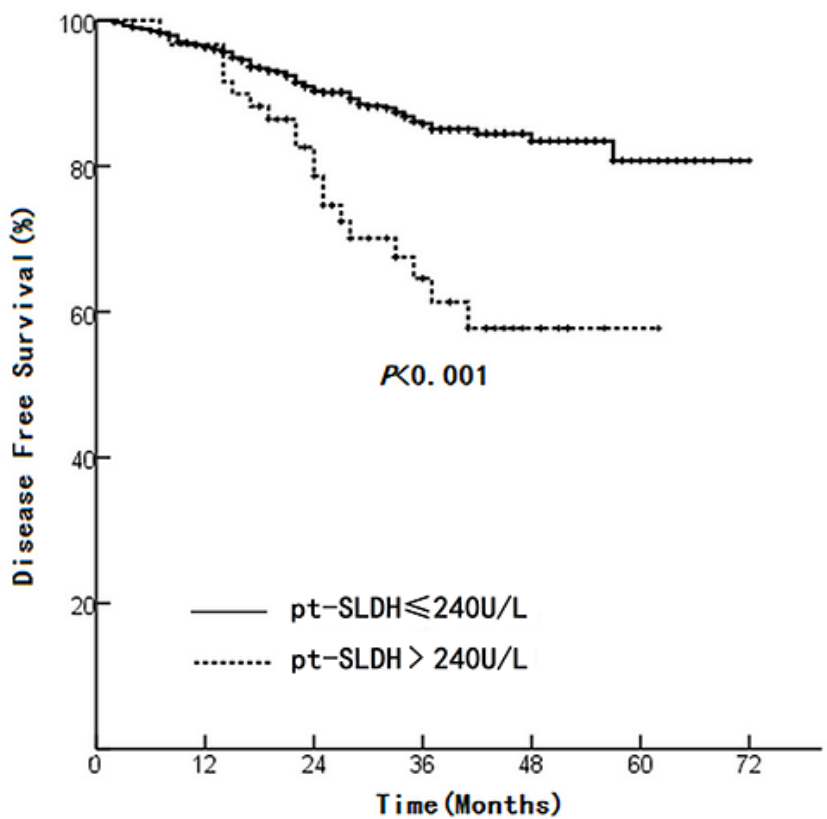

Figure 2: Comparison between high and normal pt-SLDH in (A): OS, (B): LRFS, (C): DMFS, and (D): DFS. 
study was not clear. Elevated SLDH might not only be a reflection of heavier tumor burden, but also promote tumor progression by influencing the tumor metabolism and microenvironment. $\mathrm{LDH}$ is an important enzyme in energy production under hypoxic conditions and plays critical roles in regulating carcinogenesis, tumor metabolic reprogramming, and tumor angiogenesis. The association between LDH and oncogenic anaerobic glycolysis, or the Warburg effect, could facilitate tumor growth and metastasis. This metabolic reprogramming is regulated by
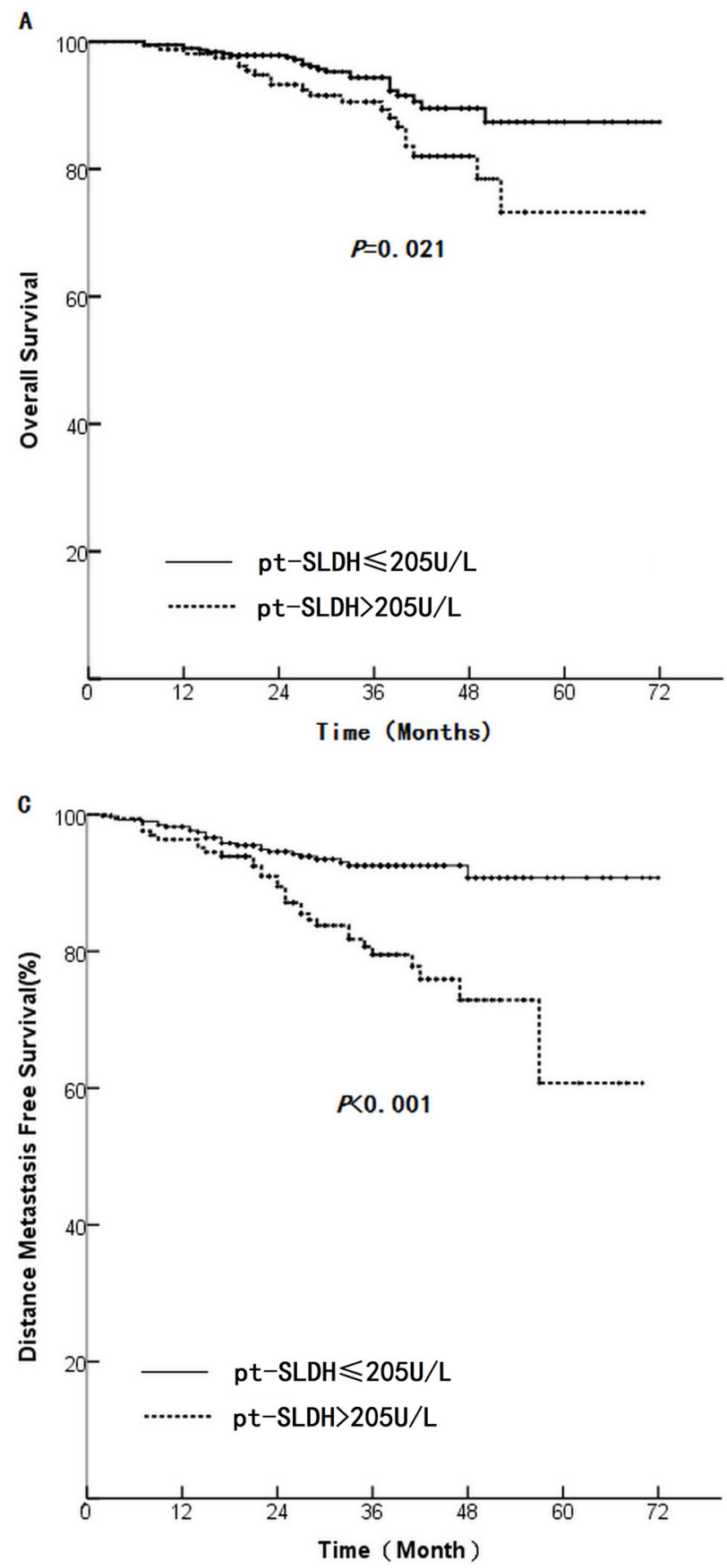

HIF-1 $\alpha$, through the transcriptional activation of key genes encoding metabolic enzymes including LDH. This process is closely associated with an increased risk of invasion, metastasis, and mortality [20, 21]. It is reasonable to speculate that high SLDH could result in poor survival through aggressive tumor progression.

Many studies have reported that age was an independent factor for prognosis of NPC, and we observed the same result $[22,23]$. The mechanism is still not clear. No correlation was found between age and pt-SLDH or
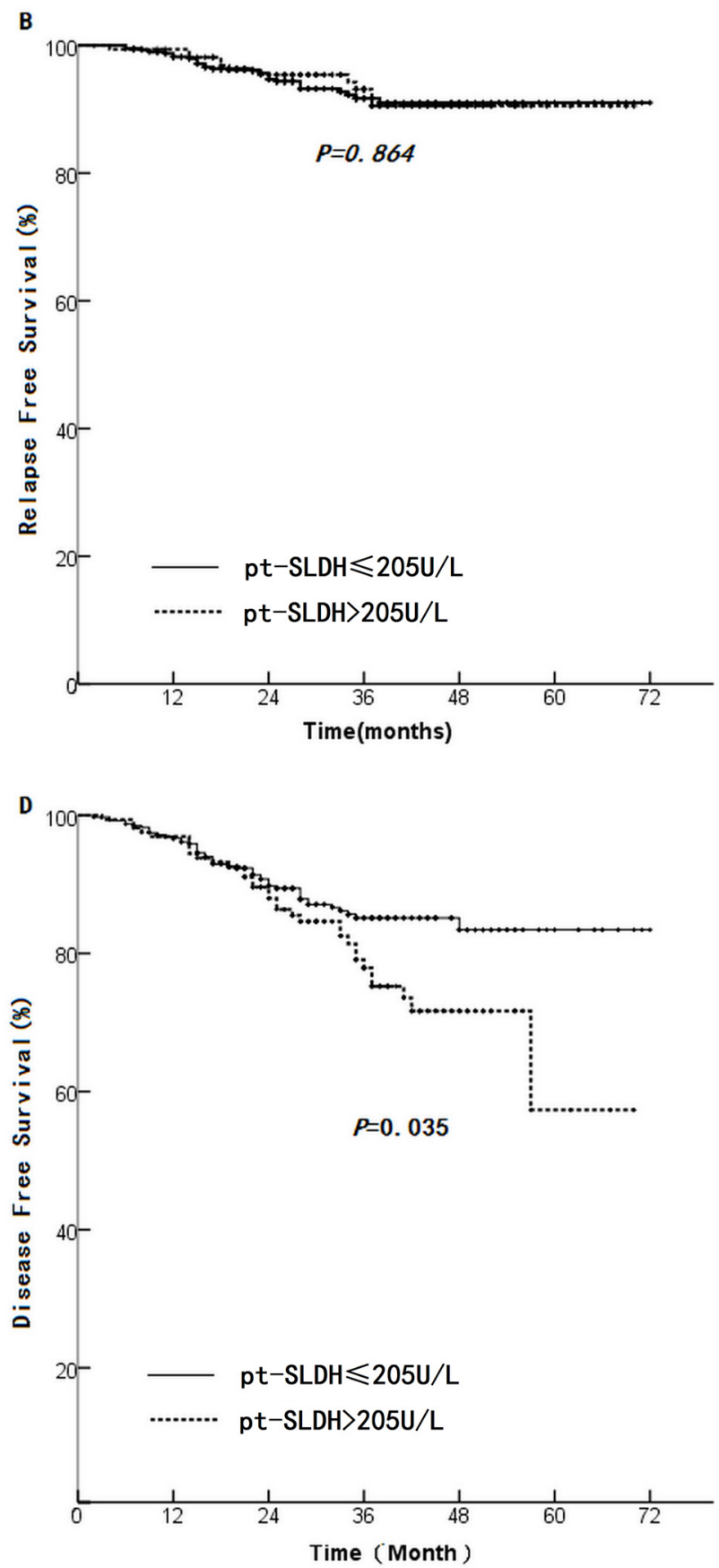

Figure 3: Comparison of survival rate between patients with pt-SLDH $>205 \mathrm{U} / \mathrm{L}$ and those with $\mathrm{pt-SLDH}<205 \mathrm{U} / \mathrm{L}$. (A): OS, (B): LRFS, (C): DMFS, and (D): DFS. 
the $\mathrm{V}_{\mathrm{SLDH}}$. Delayed diagnosis of NPC could be one of the causes, based on the observation that older patient had higher stage of malignancy.

There are certain limitations in this study. As a retrospective study, certain bias may existed in patient selection and application of RT and chemotherapy. LDH has five isoenzymes, and each plays different role in tumor growth and distant metastases. In this study, our data could not distinguish the serum level of any particular isoenzyme, which could be even more important for further investigation.

In conclusion, our finding indicated that elevated serum LDH after radiotherapy provided could be a simple available prognostic marker for distant metastasis, relapse and survival of in-situ NPC patients. In clinical practice, physician should pay more attention to those with abnormal pt-SLDH, or whose $\mathrm{V}_{\mathrm{SLDH}}$ is above 29U/L. More examination should be ordered for metastasis
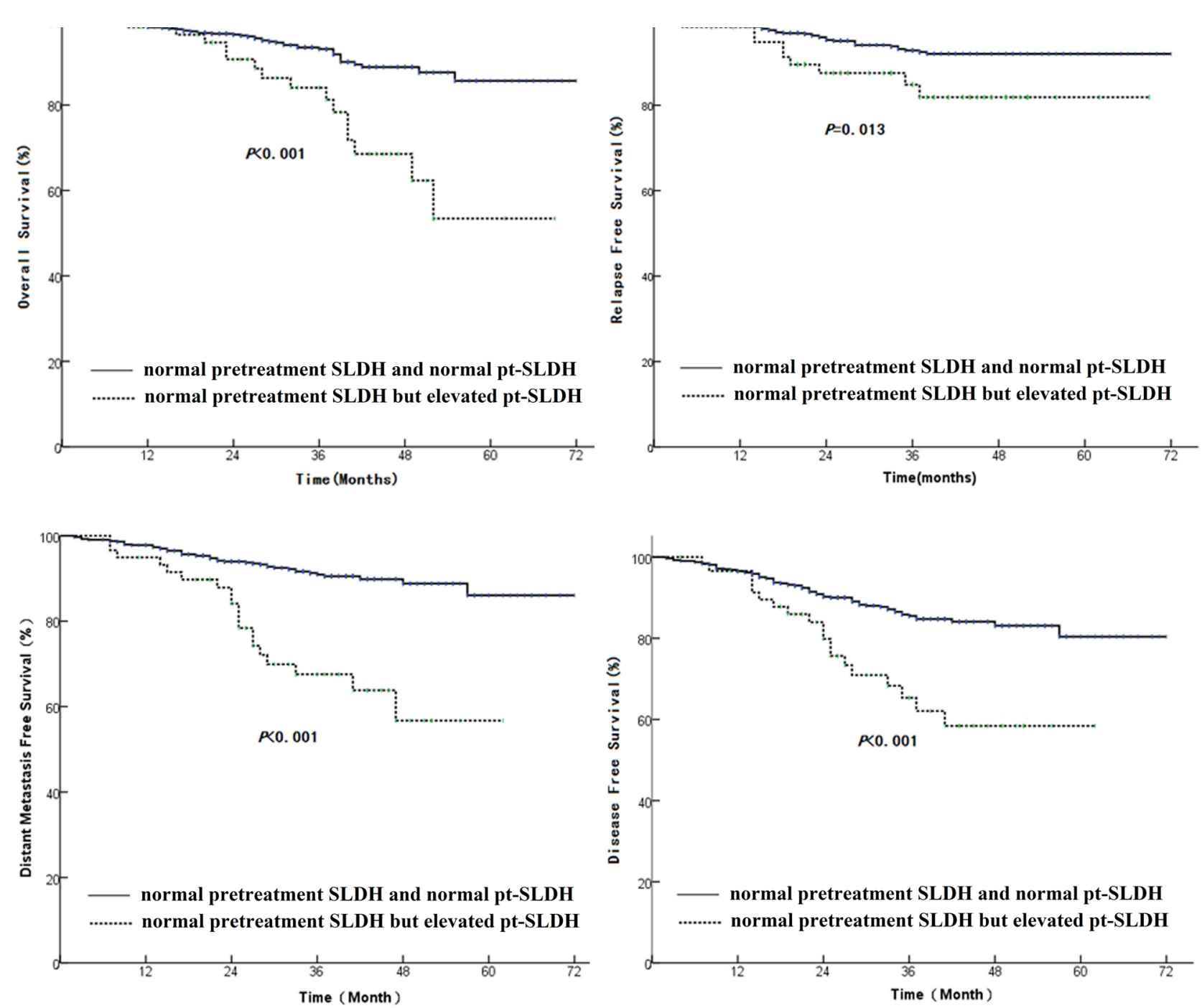

Figure 4: Comparison of survival rate between patients having normal pretreatment SLDH and normal pt-SLDH with those having normal pretreatment SLDH but elevated pt-SLDH. (A): OS, (B): LRFS, (C): DMFS, and (D): DFS.

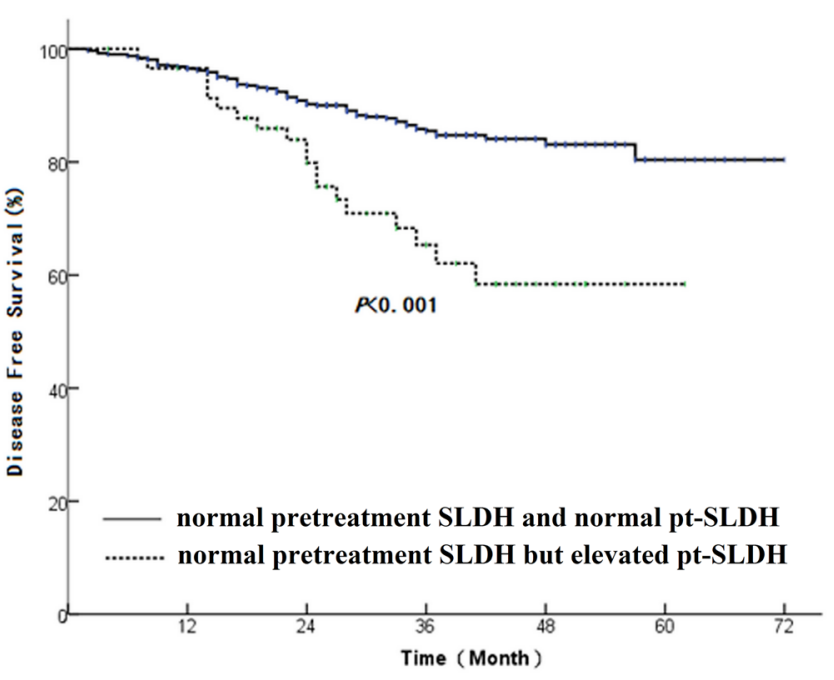

detection. Future study could focus on the identification of the isoenzyme of $\mathrm{LDH}$ and/or in combination with other tumor marker, such as EBV, for more sensitive and specific prediction $[24,25]$.

\section{MATERIALS AND METHODS}

\section{Patient}

The protocol was approved by the Institutional Review Board (IRB) of Zhejiang Cancer hospital. The NPC patient records hospitalized in Zhejiang Cancer Hospital between January 2007 and May 2012 were reviewed. Data from patients who met the following criteria were selected for further analysis: 18 or older; histologically diagnosed as stage I-IVb NPC (based on the American Joint of Cancer Committee 2009 stage system); complete pre- and post-treatment SLDH record; more than 
3 month follow- up; Karnofsky Performance Scores > 70; normal renal and cardiac function; no complications as active hepatitis, tuberculosis, acute infections, pneumonia, and pulmonary infarction during treatment and follow-up period; and has been treated with a full course of IMRT.

\section{Radiotherapy}

All patients received curative IMRT as described by Chen et al. [26, 27]. Briefly, patients were positioned in supine and immobilized from head to neck with a
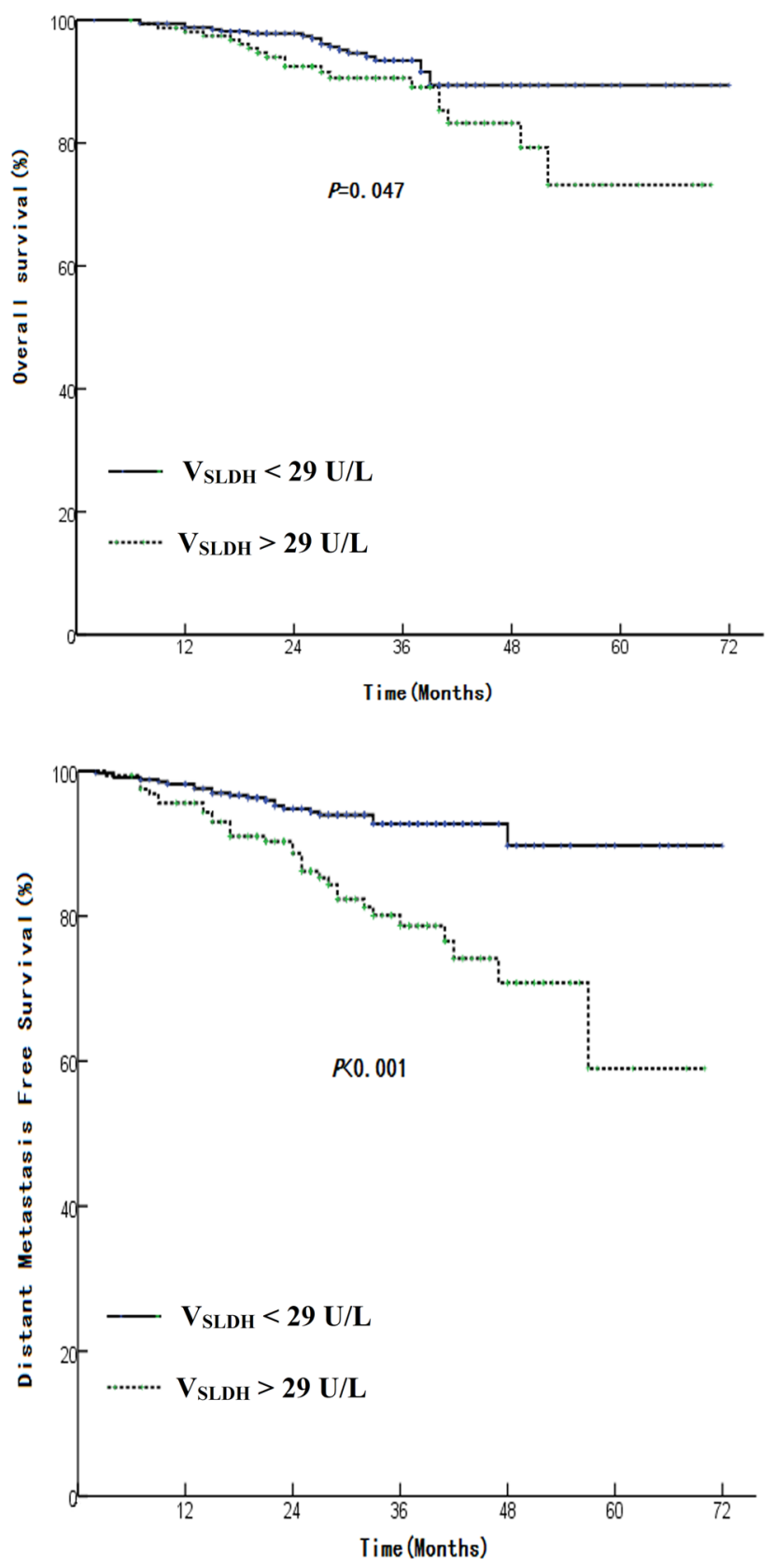

thermoplastic mask in both CT simulation and treatment delivery. The planning CT was acquired with a slice thickness of $3 \mathrm{~mm}$ and was transferred to a treatmentplanning system (PINNCAL 8.0/9.2, PHILIPS Corp.) for IMRT planning. The gross tumor volume (GTV) was defined according to diagnostic MRI, CT and physical examination. The clinical target volume 1 (CTV1) was defined as the nasopharynx GTV plus a 5 to $10 \mathrm{~mm}$ margin, while CTV2 was the CTV1 plus a 5 to $10 \mathrm{~mm}$ margin except 2-3 mm posteriorly, including the elective neck area. Planning target volumes (PTV) for all GTVs
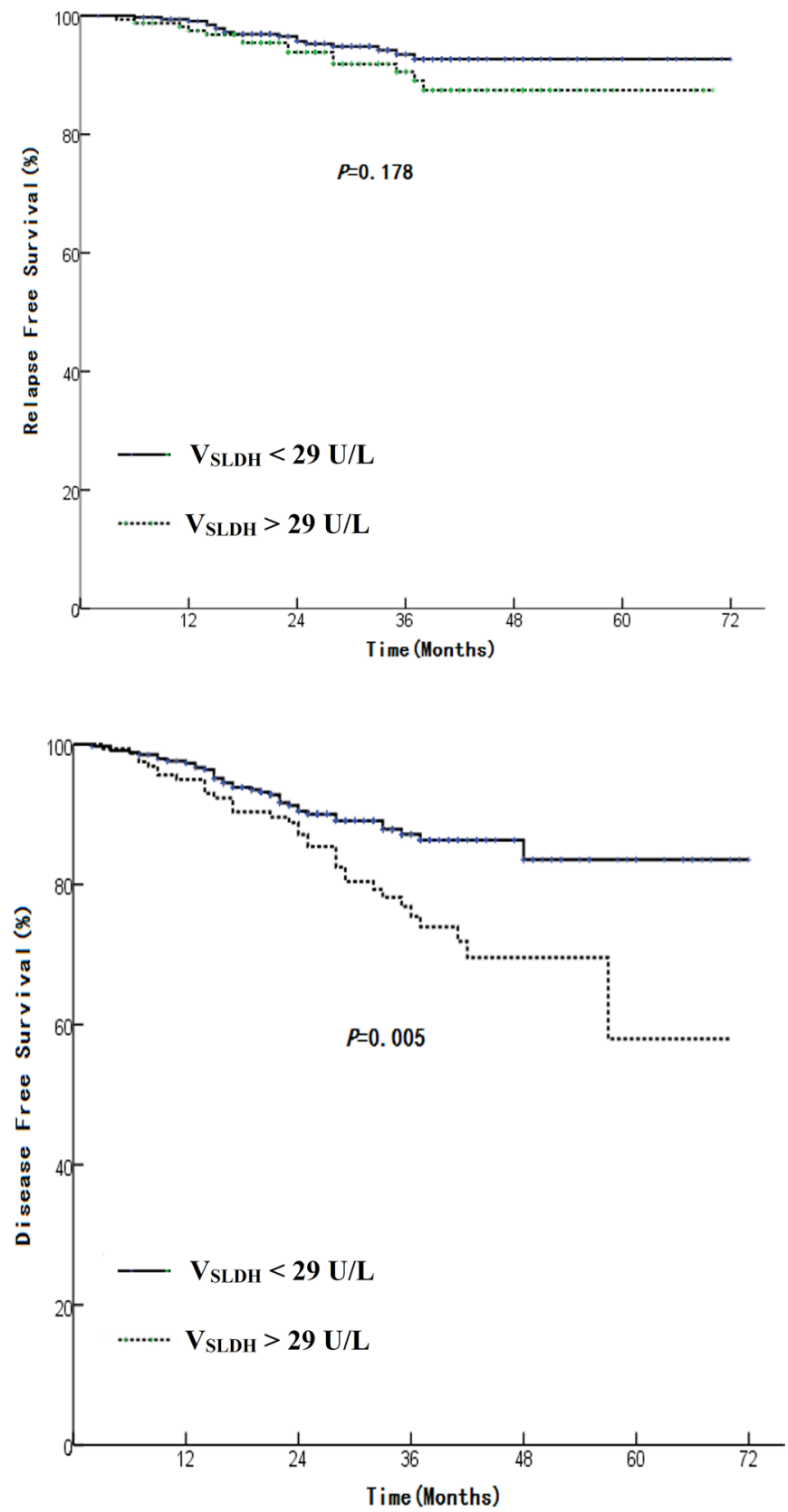

Figure 5: Comparison of survival rate between patients having a $\mathrm{V}_{\text {SLDH }}<29 \mathrm{U} / \mathrm{L}$ with those have a $\mathrm{V}_{\text {SLDH }}>29 \mathrm{U} / \mathrm{L}$. $\mathrm{V}_{\mathrm{SLDH}}=$ pt-SLDH - pretreatment SLDH. (A): OS, (B): LRFS, (C): DMFS, and (D): DFS. 
and CTVs were generated automatically by adding a non- uniform margin to account for the immobilization and localization uncertainties $(\leq 3 \mathrm{~mm})$. Organs at risk (OAR) were delineated from the planning CT. IMRT plans were generated with a prescribed dose of 69 Gy in 30 fractions to the GTV, 60-64 Gy to CTV1 and 50-54 Gy to CTV2. The doses to OARs were minimized without sacrificing target coverage.

\section{Chemotherapy}

Patients received induction chemotherapy, concurrent or adjuvant chemotherapy as described by Chen et al. [26, 27]. Briefly, the induction chemotherapy consisted of two or three cycles of docetaxel $75 \mathrm{mg} / \mathrm{m}^{2}$ and cisplatin $75 \mathrm{mg} / \mathrm{m}^{2}$ every three weeks, or cisplatin $80 \mathrm{mg} / \mathrm{m}^{2}$ on Day 1 and 5-fluorouracil $4.0 \mathrm{~g} / \mathrm{m}^{2}$ by continuous intravenous infusion on 120 hours every three weeks. For concurrent chemotherapy, cisplatin was given with $80 \mathrm{mg} / \mathrm{m}^{2}$ every three weeks or $40 \mathrm{mg} / \mathrm{m}^{2} /$ week during the course of RT. Adjuvant chemotherapy plan included cisplatin $80 \mathrm{mg} / \mathrm{m}^{2}$ on day 1 and 5-fluorouracil $4.0 \mathrm{~g} / \mathrm{m}^{2}$ by continuous intravenous infusion on 120 hours every three weeks for 3 to 4 cycles $[26,27]$.

\section{Follow-ups}

Nasopharynx was observed under indirect laryngoscopy or fiberoptic pharyngorhinoscopy for disease remission every week during RT, and 4 weeks and 12 weeks after RT. After the completion of entire treatment, patients were followed every two months in the first 6 months, every three month in the following 7 to 40 months, and once a year afterwards. The examined items in each follow up included blood routine and biochemical examination (including SLDH), chest X-ray, abdominal B type ultrasonic examination, head and neck CT or MRI, and other examinations when necessary $[26,27]$.

\section{SLDH measurement}

SLDH was measured prior to the radio- and chemotherapy and at each visit time during follow up period by Hitachi Modular 7600 Chemistry Analyzer. The optimized standard method recommended by the German Society of Clinical Chemistry was employed. A value of $>240 \mathrm{U} / \mathrm{L}$ was considered abnormal [28].

\section{Statistical analysis}

The chi-square test or Fisher's exact test (when expected count $<5$ ) was used for comparison of categorical data. K-M regression was used to determine independent factor for survival. Receiver operating characteristic curves (ROCs) were used to calculate sensitivity and specificity at each cut off value of the parameters. The optimal cut off value is achieved when the sum of the sensitivity and specificity reaches a maximum value. COX regression multivariate was performed for each of the clinical end points (OS, DMFS, LRFS, DFS) in order to evaluate the potential significant prognostic factors. All statistical tests were two-sided and a $p$ value of less than 0.05 was considered statistically significant. The data processing and statistical analyses were performed using SPSS software (Version 15.0, SPSS Inc, Chicago, IL).

\section{GRANT SUPPORT}

The study is partially supported by grants from Zhejiang Provincial Natural Science Foundation for Young Scholars (No. LQ14H160005), and the Foundation of Health and Family Planning Commission of Zhejiang Province (No. 2015KYA034).

\section{CONFLICTS OF INTEREST}

There is no conflict of interest for all authors.

\section{REFERENCES}

1. Xia P, Fu KK, Wong GW, Akazawa C, Verhey LJ. Comparison of treatment plans involving intensitymodulated radiotherapy for nasopharyngeal carcinoma. Int J Radiat Oncol Biol Phys. 2000; 48:329-337.

2. hLi AC, Xiao WW, Shen GZ, Wang L, Xu AA, Cao YQ, Huang SM, Lin CG, Han F, Deng XW, Zhao C. Distant metastasis risk and patterns of nasopharyngeal carcinoma in the era of IMRT: long-term results and benefits of chemotherapy. Oncotarget. 2015; 6:24511-24521. doi: 10.18632/oncotarget.4312.

3. Kwong D, Sham J, Choy D. The effect of loco-regional control on distant metastatic dissemination in carcinoma of the nasopharynx: an analysis of 1301 patients. Int J Radiat Oncol Biol Phys. 1994; 30:1029-1036.

4. Fang FM, Tsai WL, Chien CY, Chen HC, Hsu HC, Huang TL, Lee TF, Huang HY, Lee CH. Pretreatment quality of life as a predictor of distant metastasis and survival for patients with nasopharyngeal carcinoma. J Clin Oncol. 2010; 28:4384-4389.

5. Jin Y, Ye X, Shao L, Lin BC, He CX, Zhang BB, Zhang YP. Serum lactic dehydrogenase strongly predicts survival in metastatic nasopharyngeal carcinoma treated with palliative chemotherapy. Eur J Cancer. 2013; 49:1619-1626.

6. Motzer RJ, Mazumdar M, Bacik J, Berg W, Amsterdam A, Ferrara J. Survival and prognostic stratification of 670 patients with advanced renal cell carcinoma. J Clin Oncol. 1999; 17:2530-2540.

7. Yuce K, Baykal C, Genc C, Al A, Ayhan A. Diagnostic and prognostic value of serum and peritoneal fluid lactate dehydrogenase in epithelial ovarian cancer. Eur J Gynaecol Oncol. 2001; 22:228-232. 
8. Cheng SH, Jian JJ, Tsai SY, Chan KY, Yen LK, Chu NM, Tan TD, Tsou MH, Huang AT. Prognostic features and treatment outcome in locoregionally advanced nasopharyngeal carcinoma following concurrent chemotherapy and radiotherapy. Int J Radiat Oncol Biol Phys. 1998; 41:755-762.

9. Turen S, Ozyar E, Altundag K, Gullu I, Atahan IL. Serum lactate dehydrogenase level is a prognostic factor in patients with locoregionally advanced nasopharyngeal carcinoma treated with chemoradiotherapy. Cancer Invest. 2007; 25:315-321.

10. Liaw CC, Wang CH, Huang JS, Kiu MC, Chen JS, Chang HK. Serum lactate dehydrogenase level in patients with nasopharyngeal carcinoma. Acta oncologica. 1997; 36:159-164.

11. Tian YM, Zeng L, Wang FH, Liu S, Guan Y, Lu TX, Han F. Prognostic factors in nasopharyngeal carcinoma with synchronous liver metastasis: a retrospective study for the management of treatment. Radiation oncology. 2013; 8:272.

12. Cheng SH, Tsai SY, Horng CF, Yen KL, Jian JJ, Chan KY, Lin CY, Terng SD, Tsou MH, Chu NM, Chen HH, Chen PL, Chung YL, et al. A prognostic scoring system for locoregional control in nasopharyngeal carcinoma following conformal radiotherapy. Int J Radiat Oncol Biol Phys. 2006; 66:992-1003.

13. Caglar M, Ceylan E, Ozyar E. Frequency of skeletal metastases in nasopharyngeal carcinoma after initiation of therapy: should bone scans be used for follow-up? Nucl Med Commun. 2003; 24:1231-1236.

14. Zen HG, Jame JM, Chang AY, Li WY, Law CK, Chen KY, Lin CZ. Nasopharyngeal carcinoma with bone marrow metastasis. Am J Clin Oncol. 1991; 14:66-70.

15. Hong RL, Ting LL, Ko JY, Hsu MM, Sheen TS, Lou PJ, Wang CC, Chung NN, Lui LT. Induction chemotherapy with mitomycin, epirubicin, cisplatin, fluorouracil, and leucovorin followed by radiotherapy in the treatment of locoregionally advanced nasopharyngeal carcinoma. J Clin Oncol. 2001; 19:4305-4313.

16. Zhou GQ, Tang LL, Mao YP, Chen L, Li WF, Sun Y, Liu LZ, Li L, Lin AH, Ma J. Baseline serum lactate dehydrogenase levels for patients treated with intensitymodulated radiotherapy for nasopharyngeal carcinoma: a predictor of poor prognosis and subsequent liver metastasis. Int J Radiat Oncol Biol Phys. 2012; 82:e359-365.

17. Wan XB, Wei L, Li H, Dong M, Lin Q, Ma XK, Huang PY, Wen JY, Li X, Chen J, Ruan DY, Lin ZX, Chen ZH, et al. High pretreatment serum lactate dehydrogenase level correlates with disease relapse and predicts an inferior outcome in locally advanced nasopharyngeal carcinoma. Eur J Cancer. 2013; 49:2356-2364.

18. Yang Y, Su D, Zhao L, Zhang D, Xu J, Wan J, Fan S, Chen M. Different effects of LDH-A inhibition by oxamate in non-small cell lung cancer cells. Oncotarget. 2014; 5:11886-11896. doi: 10.18632/oncotarget.2620.
19. Zhai X, Yang Y, Wan J, Zhu R, Wu Y. Inhibition of LDH-A by oxamate induces G2/M arrest, apoptosis and increases radiosensitivity in nasopharyngeal carcinoma cells. Oncol Rep. 2013; 30:2983-2991.

20. Zhang W, Chen Y, Zhou G, Liu X, Chen L, Tang L, Mao Y, Sun Y, Ma J. Pretreatment Serum Lactate Dehydrogenase and N Classification Predict Long-Term Survival and Distant Metastasis in Patients With Nasopharyngeal Carcinoma Who Have A Positive Family History of Cancer. Medicine (Baltimore). 2015; 94:e1505.

21. Petrelli F, Cabiddu M, Coinu A, Borgonovo K, Ghilardi M, Lonati V, Barni S. Prognostic role of lactate dehydrogenase in solid tumors: a systematic review and meta-analysis of 76 studies. Acta oncologica. 2015; 54:961-970.

22. Mak HW, Lee SH, Chee J, Tham I, Goh BC, Chao SS, Ong YK, Loh KS, Lim CM. Clinical Outcome among Nasopharyngeal Cancer Patients in a Multi-Ethnic Society in Singapore. PloS one. 2015; 10:e0126108.

23. Xiao G, Cao Y, Qiu X, Wang W, Wang Y. Influence of gender and age on the survival of patients with nasopharyngeal carcinoma. BMC cancer. 2013; 13:226.

24. Lynn TC, Hsieh RP, Chuang CY, Huang SC, Hsieh T, Tu SM. Epstein-Barr virus-associated antibodies and serum biochemistry in nasopharyngeal carcinoma. Laryngoscope. 1984; 94:1485-1488.

25. Cheng SH, Yen KL, Jian JJ, Tsai SY, Chu NM, Leu SY, Chan KY, Tan TD, Cheng JC, Hsieh CY, Huang AT. Examining prognostic factors and patterns of failure in nasopharyngeal carcinoma following concomitant radiotherapy and chemotherapy: impact on future clinical trials. Int J Radiat Oncol Biol Phys. 2001; 50:717-726.

26. Chen YY, Zhao C, Wang J, Ma HL, Lai SZ, Liu Y, Han F, Lu LX, Bao Y, Chen M. Intensity-modulated radiation therapy reduces radiation-induced trismus in patients with nasopharyngeal carcinoma: a prospective study with $>5$ years of follow-up. Cancer. 2011; 117:2910-2916.

27. Wang J, Chen YY, Tai A, Chen XL, Huang SM, Yang C, Bao Y, Li NW, Deng XW, Zhao C, Chen M, Li XA. Sensorineural Hearing Loss after Combined Intensity Modulated Radiation Therapy and Cisplatin-Based Chemotherapy for Nasopharyngeal Carcinoma. Translational oncology. 2015; 8:456-462.

28. Jurisic V, Radenkovic S, Konjevic G. The Actual Role of $\mathrm{LDH}$ as Tumor Marker, Biochemical and Clinical Aspects. Advances in experimental medicine and biology. 2015; 867:115-124. 